\title{
OPTIMAL DRIVING STRATEGIES FOR A TRAIN JOURNEY WITH SPEED LIMITS
}

\author{
PETER PUDNEY and PHIL HOWLETT ${ }^{1}$
}

(Received 31 August 1992; revised 25 November 1992)

\begin{abstract}
How should a vehicle be driven to minimise fuel consumption? In this paper we consider the case where a train is to be driven along a straight, level track, but where speed limits may apply over parts of the track. The journey is to be completed within a specified time using as little fuel as possible.

For a journey without speed limits, the optimal driving strategy typically requires full power, speed holding, coasting and full braking, in that order. The holding speed and braking speed can be determined from the vehicle characteristics and the time available to complete the journey. If the vehicle has discrete control settings, the holding phase should be approximated by alternate coast and power phases between two critical speeds.

For a journey with speed limits, a similar strategy applies. For each given journey time there is a unique holding speed. On intervals of track where the speed limit is below the desired holding speed, the speed must be held at the limit. If braking is necessary on an interval, the speed at which braking commences is determined in part by the holding speed for the interval. For vehicles with discrete control, speed-holding is approximated by alternate coast and power phases between two critical speeds, or between a lower critical speed and the speed limit.
\end{abstract}

\section{Introduction}

Systems for calculating efficient driving advice during a journey are particularly applicable to timetabled suburban and long-haul railway journeys. The Metromiser [2] is an example of a system that displays efficient driving advice to the driver of a suburban train. It is easy to use, and has achieved fuel savings in excess of $20 \%$ and significant improvements in timekeeping. It does not, however, generate optimal advice for long journey segments, journey segments with many changes of gradient, or journey segments with multiple restrictive speed limits.

In this paper we will consider the case where a train is to be driven along a straight,

\footnotetext{
'Scheduling and Control Group, University of South Australia, Adelaide, Australia

(C) Australian Mathematical Society, 1994, Serial-fee code 0334-2700/94
} 
level track of fixed length, but where speed limits apply over various track intervals. The journey is to be completed within a specified time, using as little fuel as possible.

The train control problem has been considered by various authors. Milroy [9] used an heuristic application of the Pontryagin Principle to obtain conditions for a journey that minimised the mechanical energy supplied during tractive acceleration. His results were confirmed independently by Kraft and Schnieder [8], Asnis et al. [1] and by Howlett [4].

We will approach the problem by considering a vehicle with discrete control settings, and by considering journeys with a prescribed control sequence. The problem then becomes one of determining where the control should be changed. Once we determine necessary conditions on the optimal switching locations for a given sequence, we can then consider the problem of determining which sequence is optimal.

A similar approach has been used by Cheng and Howlett $[7,6]$ for the case where the vehicle is to be driven over level track without speed limits. Recently this work has been extended to the case of an undulating track [5, 3]. The extended procedure may also be applicable to curved tracks. By combining this work, we hope to develop a method for calculating optimal driving strategies for curved, undulating tracks with speed limits. Ultimately we wish to develop a vehicle-borne system, suited to a variety of vehicles, that can monitor the progress of a journey and recalculate the appropriate optimal driving strategy from any given point on the journey. Such a device would be able to compensate for the inevitable departures from any pre-planned optimal strategy.

\section{Vehicle model}

Assume that the vehicle has a number of discrete control settings denoted by

$$
u \in U=\{-q, \ldots,-2,-1,0,1,2, \ldots, Q\},
$$

where $q \quad$ is the number of brake control settings,

$Q \quad$ is the number of traction control settings,

$u=0$ represents coasting.

On straight, level track the acceleration of the vehicle is given by

$$
v \frac{d v}{d x}=\phi_{u}(v)-r(v)
$$

where $v \quad$ is the speed of the vehicle,

$x \quad$ is the distance travelled,

$\phi_{u}(v)$ is the tractive or braking acceleration developed by the vehicle for control setting $u$ at speed $v$,

$r(v)$ is the deceleration of the vehicle due to resistance at speed $v$. 
We assume a constant cost rate $c_{u}$ for each control setting, and that $c_{u}=0$ for $u \leq 0$. We also assume that $\phi_{u}(v)$ decreases as $v$ increases, and that $r(v)$ increases as $v$ increases.

\section{Journey model}

Consider a journey with $n$ distinct phases. The control setting is specified for each phase. The distances at which the control must be changed are to be determined. These distances are denoted by $0=x_{0}<x_{1}<\ldots<x_{n}=X$. The journey starts at distance $x=0$ and finishes at distance $x=X$.

The track speed limit changes at distances $0=X_{0}<X_{1}<\ldots<X_{p}=X$. The speed limit for the interval $\left(X_{j}, X_{j+1}\right)$ is $M_{j+1}$.

It is necessary to determine the locations of the points $\left\{x_{k}\right\}$ for $k=1,2, \ldots, n-1$ in relation to the fixed points $\left\{X_{j}\right\}$ for $j=0,1, \ldots, p$. We use the notation $\{r(j)\}$ to denote the sequence with $r(0)=0$ and $r(p)=n$ and with

$$
x_{r(j)} \leq X_{j}<x_{r(j)+1} \quad j=1,2, \ldots, p-1 .
$$

Let $\xi_{k+1}=x_{k+1}-x_{k}$ denote the length of the interval $\left[x_{k}, x_{k+1}\right]$. The control setting in this interval is $u(k+1)$. If the time taken to traverse the interval $\left[x_{k}, x_{k+1}\right]$ is $\tau_{k+1}$ then the cost of the journey is

$$
J=\sum_{k=0}^{n-1} c_{u(k+1)} \tau_{k+1}
$$

If the time allowed for the journey is $T$, we require

$$
\sum_{k=0}^{n-1} \tau_{k+1} \leq T
$$

The speed of the vehicle at the beginning of the journey is $V_{0}$. The speed of the vehicle at the end of the journey is $V_{f}$. Both speeds are given. The speed $V_{k}$ at location $x_{k}$ must be determined for $k=1,2, \ldots, n-1$. It can be shown that $V_{k}$ depends only on $\xi_{1}, \ldots, \xi_{k}$.

\section{Fundamental speed profiles}

Following Howlett [4], we can define distance as a function of speed by

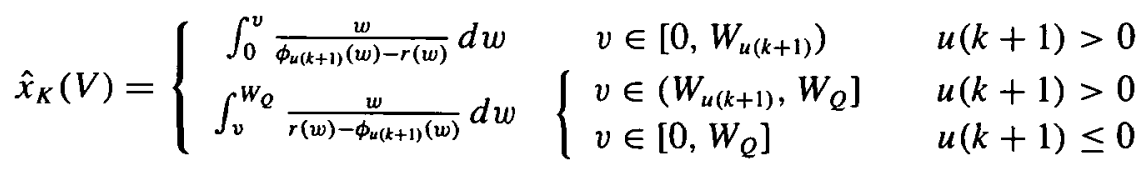


where $W_{u}$ is the limiting speed for control setting $u$.

If $\phi_{u}(0) \geq r(0)$, the limiting speed $W_{u}$ is the unique number given by $\phi_{u}\left(W_{u}\right)=$ $r\left(W_{u}\right)$. If we assume that $d \phi_{u}(v)<0$ when $d v<0$ then we can use (4) to show that $\hat{x}_{k}(v) \rightarrow \infty$ as $v \rightarrow W_{u}(k+1)$. In this case we have $V_{k}<W_{u(k+1)} \Rightarrow V_{k+1}<W_{u(k+1)}$ and $V_{k}>W_{u(k+1)} \Rightarrow V_{k+1}>W_{u(k+1)}$. The monotonic nature of the speed on $\left[x_{k}, x_{k+1}\right]$ means that speed limits can be imposed on the entire interval by constraining $V_{k}$ and $V_{k+1}$.

If $\phi_{u}(0)<r(0)$ then we define $W_{u}=0$.

The function $\hat{v}_{k}$ is the inverse of $\hat{x}_{k}$. The actual speed on the interval $\left[x_{k}, x_{k+1}\right]$ is given by

$$
v(x)=\hat{v}_{k}\left(x-x_{k}+\hat{x}_{k}\left(V_{k}\right)\right)
$$

provided that $V_{k} \neq W_{u(k+1)}$.

The speed $U_{j}$ at location $X_{j}$ for $j=1,2, \ldots, p-1$ is

$$
U_{j}=\hat{v}_{r(j)}\left(X_{j}-x_{r(j)}+\hat{x}_{r(j)}\left(V_{r(j)}\right)\right) .
$$

The time required to traverse the interval $\left[x_{k}, x_{k+1}\right]$ is given by

$$
\tau_{k+1}=\int_{0}^{\xi_{k+1}} \frac{1}{\hat{v}_{k}\left(\xi+\hat{x}_{k}\left(V_{k}\right)\right)} d \xi
$$

\section{Optimal driving strategies}

Since $V_{k}=V_{k}\left(\xi_{1}, \xi_{2}, \ldots, \xi_{k}\right)$, we have $\tau_{k}=\tau_{k}\left(\xi_{1}, \xi_{2}, \ldots, \xi_{k}\right)$. If we write $\xi=$ $\left(\xi_{1}, \xi_{2}, \ldots, \xi_{n}\right) \in \mathscr{R}^{n}$ then the key variables can be written as functions of $\xi$.

The cost of the journey is

$$
J(\xi)=\sum_{k=0}^{n-1} c_{u(k+1)} \tau_{k+1},
$$

the total distance travelled is

$$
\Theta(\xi)=\sum_{k=0}^{n-1} \xi_{k},
$$

and the time taken for the journey is

$$
T(\xi)=\sum_{k=0}^{n-1} \tau_{k+1} .
$$

We wish to minimise the cost of the journey $J(\xi)$. We require that the total distance travelled and the total time taken satisfy the constraints

$$
\Theta(\xi)=X, \quad T(\xi) \leq T .
$$


Note that the constraint $\Theta(\xi)=X$ can be weakened to read $\Theta(\xi) \geq X$ with no change to the solution.

In addition to the distance and time constraints, we must also satisfy the speed constraints

$$
\begin{aligned}
& V_{K+1} \leq M_{j} \quad \text { where } \quad r(j-1)<k+1<r(j) \quad k=0,1, \ldots, n-2 \text {, } \\
& U_{j} \leq N_{j} \quad \text { where } \quad j=1,2, \ldots, p-1 \text {, }
\end{aligned}
$$

where $N_{j}$ is the most restrictive of the speed limits at $x=X_{j}$, i.e. $N_{j}=\min \left\{M_{j}, M_{j+1}\right\}$. The first of these constraints ensures that the switching speeds do not exceed the speed limit on any interval $\left(X_{j}, X_{j+1}\right)$. This is sufficient to ensure that the vehicle speed will not exceed the speed limit, since the speed of the vehicle will always lie between the switching speeds. The second ensures that speed limits are not exceeded at any point $X_{j}$ where the speed limit changes. We ensure that the journey finishes at the correct speed by adding the constraint $V_{n}=V_{f}$. We define a Lagrangean function

$$
\begin{aligned}
\mathscr{J}(\xi)= & J(\xi)+\lambda[X-\Theta(\xi)]+\mu[t(\xi)-T]+\sum_{j=1}^{p-1} \sum_{k=r(j-1)}^{r(j)-1} \rho_{k+1}\left[V_{k+1}-M_{j}\right] \\
& +\sum_{k=r(p-1)}^{r(p)-2} \rho_{k+1}\left[V_{k+1}-M_{p}\right]+\sigma\left[V_{n}-V_{f}\right]+\sum_{j=1}^{p-1} \eta_{j}\left[U_{j}-N_{j}\right]
\end{aligned}
$$

where $\lambda, \mu \in \mathscr{R}^{n}, \rho=\left(\rho_{1}, \rho_{2}, \ldots, \rho_{n-1}\right) \in \mathscr{R}^{n-1}, \sigma \in \mathscr{R}$ and $\eta=\left(\eta_{1}, \eta_{2}, \ldots, \eta_{p-1}\right)$ $\in \mathscr{R}^{p-1}$.

We then apply the Kuhn-Tucker conditions

$$
\frac{\partial \mathscr{J}}{\partial \xi_{k+1}}=0 \quad k=0,1, \ldots, n-1
$$

and the complementary slackness conditions

$$
\begin{array}{rlrl}
\lambda[X-\Theta(\xi) & =0 & & \lambda \geq 0 \\
\mu[t(\xi)-T]=0 & & \mu \geq 0 & \\
\rho_{k+1}\left[V_{k+1}-M_{j}\right]=0 & & \rho_{k+1} \geq 0 & r(j-1)<k+1 \leq r(j+1) \\
& & j=1,2, \ldots, p-1 \\
\sigma\left[V_{n}-V_{f}\right]=0 & & \\
\eta_{j}\left[U_{j}-N_{j}\right]=0 & & \eta_{j} \geq 0 & j=1,2, \ldots, p-1,
\end{array}
$$

and assume that $\xi$ lies in an open set such that $V_{k}(\xi) \neq W_{u(k)}$ for all $k$. 
For $k=0,1, \ldots, n-1$ and $j$ such that $r(j-1)<k+1 \leq r(j)$

$$
\begin{aligned}
\frac{\partial \mathscr{J}}{\partial \xi_{k+1}}=\sum_{h=k}^{n-1} c_{u(h+1)} \frac{\partial \tau_{h+1}}{\partial \xi_{k+1}}-\lambda+\mu \sum_{h=k}^{n-1} \frac{\partial \tau_{h+1}}{\partial \xi_{k+1}} & +\sum_{h=k+1}^{n-2} \rho_{h+1} \frac{\partial V_{h+1}}{\partial \xi_{k+1}} \\
& +\sigma \frac{\partial V_{n}}{\partial \xi_{k+1}}+\sum_{l=j}^{p-1} \eta_{l} \frac{\partial U_{l}}{\partial \xi_{k+1}} .
\end{aligned}
$$

The calculation of these derivatives is similar to the calculation of the corresponding derivatives in Cheng and Howlett $[7,6]$.

At the end of the journey, the equation $V_{n} \partial \mathscr{J} / \partial \xi_{n}=0$ gives

$$
\lambda V_{n}-\mu=c_{u(n)}-\sigma\left[\phi_{u(N)}\left(V_{n}\right)-r\left(V_{n}\right)\right] .
$$

Since $V_{n}=V_{f}$ is known, equation (12) simply relates the three Lagrange multipliers $\lambda, \mu$ and $\sigma$.

For $k=1,2, \ldots, n-1$, the equation

$$
V_{k}\left(\frac{\partial \mathscr{J}}{\partial \xi_{k}}-R_{k}\left(V_{k}\right) \frac{\partial \mathscr{J}}{\partial \xi_{k+1}}\right)=0
$$

gives, for $j$ such that $r(j-1)<k \leq r(j)$,

$$
\begin{aligned}
\left(\sum_{l=j}^{p-1} \eta_{l} \frac{\phi_{u(r(l)+1)}\left(U_{l}\right)-r\left(U_{l}\right)}{U_{l}}+\lambda\right) V_{k}-\mu \\
=\frac{c_{u(k)}-R_{k}\left(V_{k}\right) c_{u(k+1)}}{1-R_{k}\left(V_{k}\right)}+\frac{\rho_{k} \phi_{u(k)}\left(V_{k}\right)-r\left(V_{k}\right)}{1-R_{k}\left(V_{k}\right)},
\end{aligned}
$$

where

$$
R_{s}(V)=\frac{\phi_{u(s)}(V)-r(V)}{\phi_{u(s+1)}(V)-r(V)}
$$

If we let

$$
\lambda_{j}=\left\{\begin{array}{cl}
\lambda & j=p \\
\sum_{l=j}^{p-1} \eta_{l} \frac{\phi_{u(r(l)+1)}\left(U_{l}\right)-r\left(U_{l}\right)}{U_{l}}+\lambda & j<p,
\end{array}\right.
$$

then we have $V_{k}=M_{j}$ or $\rho_{k}=0$. In the case $\rho_{k}=0$ we have

$$
\lambda_{j} V_{k}-\mu=\frac{c_{u(k)}-R_{k}\left(V_{k}\right) c_{u(k+1)}}{1-R_{k}\left(V_{k}\right)}
$$

If $\eta_{j} \neq 0$, the complementary slackness conditions require that $U_{j}=N_{j}$. If, on the other hand, $\eta_{j}=0$ and $U_{j}<N_{j}$, then $\lambda_{j}=\lambda_{j+1}$. If $U_{j}<N_{j}$ for all $j$ then $\lambda_{j}=\lambda$ and the conditions are identical to the conditions obtained by Cheng and Howlett for the case where there are no speed limits. 


\section{Critical speeds}

Consider a vehicle with three control settings: power, coast and brake. Acceleration due to the vehicle is given by

$$
\phi_{u}(v)=\left\{\begin{array}{rll}
\phi(v) & u=1 & \text { (power) } \\
0 & u=0 & \text { (coast) } \\
-B & u=-1 & \text { (brake) }
\end{array}\right.
$$

where $\phi$ is the acceleration corresponding to full tractive effort,

$B$ is the deceleration corresponding to full braking effort.

We can assume that the cost rate $c_{1}=1$ for power, and that $c_{u}$ is zero for coast and brake.

By alternating between coast and power, and by braking when necessary, the three control modes can be used to follow any possible speed profile. We will further assume that the control is never changed between power and brake without an intermediate coast phase.

When changing between coast and power, the conditions for an optimal journey require $V_{k}=M_{j}$ or $\rho_{k}=0$. In the case $\rho_{k}=0$ we get

$$
\lambda_{j} V_{k}-\mu=\frac{r\left(V_{k}\right)}{\phi\left(V_{k}\right)} .
$$

The function $F(v)=r(v) / \phi(v)$ is often strictly convex with $F(0) \geq 0$. Suburban and long-haul trains typically have $\Psi(v)=H / v$, where $H$ is the tractive power per unit mass, and $r(v)=r_{0}+r_{1} v+r_{2} V^{2}$. In such cases, (15) will generally have two solutions, $V_{k}=\mathscr{V}_{j}$ and $V_{k}=\mathscr{W}_{j}$, where $0 \leq \mathscr{V}_{j} \leq \mathscr{W}_{j}$. Furthermore, for $\lambda_{j}>\lambda_{k}$ we have $0 \leq \mathscr{V}_{j}<\mathscr{V}_{k} \leq \mathscr{W}_{k}<\mathscr{W}_{j}$. When changing between coast and brake, the conditions for an optimal journey require $V_{k}=M_{j}$ or $\rho_{k}=0$. In the case $\rho_{k}=0$, we get

$$
\lambda_{j} V_{k}-\mu=0 .
$$

Equation (16) will have one solution, $V_{k}=\mathscr{U}_{j}$, where $0 \leq \mathscr{U}_{j} \leq \mathscr{V}_{j} \leq \mathscr{W}_{j}$. The speeds $\mathscr{U}_{j}, \mathscr{V}_{j}$ and $\mathscr{W}_{j}$ are critical speeds for the interval $\left(X_{j-1}, X_{j}\right)$.

In an interval $\left(X_{j-1}, X_{j}\right)$ with speed limit $M_{j}$, the control may be changed from coast to power only when $V_{k}=\mathscr{V}_{j}$, and requires $\mathscr{V}_{j}<M_{j}$. Similarly, the control may be changed from power to coast only when $V_{k}=\min \left\{\mathscr{W}_{j}, M_{j}\right\}$. A change from coast to brake may occur when $V_{k}=\mathscr{U}_{j}$. Once braking has commenced, the speed of the vehicle will remain below the critical speeds for the interval, and so the control cannot be changed again within the interval.

At a boundary $X_{j}$ between two intervals, a change from power to coast may occur if $V_{k}=\mathscr{W}_{j}=\mathscr{W}_{j+1} \leq N_{j}$ or if $V_{k}=N_{j} \leq \min \left\{\mathscr{W}_{j}, \mathscr{W}_{j+1}\right\}$. A change from coast to 
power can occur at this point only if $V_{k}=\mathscr{V}_{j}=\mathscr{V}_{j+1}<N_{j}$. A change from brake to coast can occur if $N_{j}<\mathscr{U}_{j}$. These changes allow us to maintain a feasible strategy.

Thus the possible control changes are:

in the interval $\left(X_{j-1}, X_{j}\right)$ power to coast if $V_{k}=\min \left\{\mathscr{W}_{j}, M_{j}\right\}$

coast to power if $V_{k}=\mathscr{V}_{j}$

coast to brake if $V_{k}=\mathscr{U}_{j}$

at $X_{j}$

$$
\begin{array}{ll}
\text { power to coast } & \text { if } V_{k}=\mathscr{W}_{j}=\mathscr{W}_{j+1} \leq N_{j} \\
& \text { or } V_{k}=N_{j} \leq \min \left\{\mathscr{W}_{j}, \mathscr{W}_{j+1}\right\} \\
\text { coast to power } & \text { if } V_{k}=\mathscr{V}_{j}=\mathscr{V}_{j+1}<N_{j} \\
\text { brake to coast } & \text { if } V_{k}=N_{j} \leq \mathscr{U}_{j} .
\end{array}
$$

The construction of an optimal journey is illustrated in the diagram below.

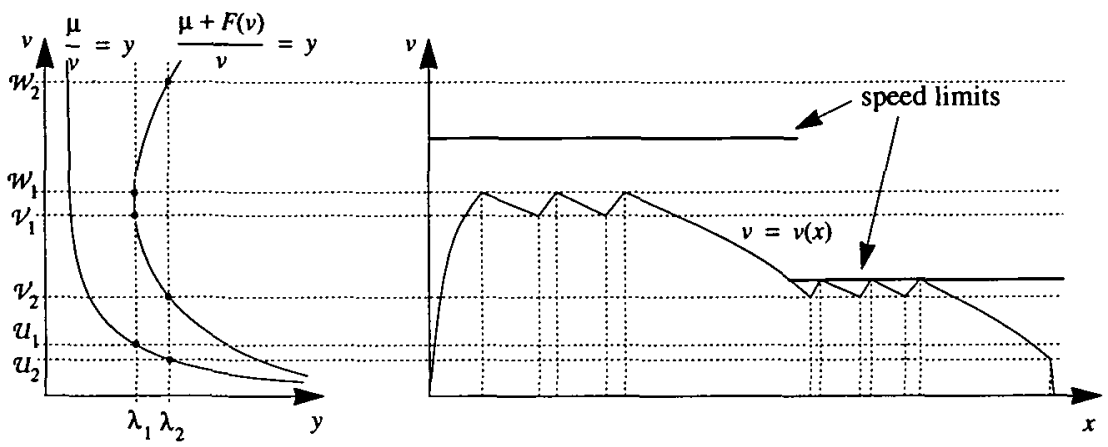

If $F(v)$ is strictly convex and if $F(v) / v \rightarrow \infty$ as $v \rightarrow \infty$, then for $\mu$ such that $\mu+F(0)>0$ it can be shown that the function $[\mu+F(v)] / v$ has a unique minimum turning point in the region $v>0$. For values of $\lambda$ above the minimum there are precisely two solutions to the equation $\lambda=[\mu+F(v)] / v$ in the region $v>0$.

The Lagrange multipliers $\mu$ and $\lambda_{j}$ determine critical speeds $\mathscr{U}_{j}, \mathscr{V}_{j}$ and $\mathscr{W}_{j}$ for each track interval $\left(X_{j}, X_{j+1}\right)$. We can construct an approximate speed-holding phase on a track interval using coast-power pairs, with the speed of the vehicle oscillating between $\mathscr{V}_{j}<M_{j}$ and $V=\min \left\{\mathscr{W}_{j}, M_{j}\right\}$. If the number of coast-power pairs is given for each track interval, then for each $\mu$ we can adjust the Lagrange multipliers $\lambda_{j}=\lambda_{j}(\mu)$ so that the distance and speed constraints are satisfied for each interval. We can then adjust $\mu$ to satisfy the time constraint for the journey.

As we increase the number of coast-power pairs, either $\mathscr{V}_{j}, \mathscr{W}_{j} \rightarrow \mathscr{V}$ where $\mathscr{V}$ is a unique holding speed for the journey, or $\mathscr{V}_{j} \rightarrow M_{j}$. Thus there is a unique holding speed for the journey. On track intervals where this holding speed is above the limit, the speed of the vehicle must be held at the limit. 


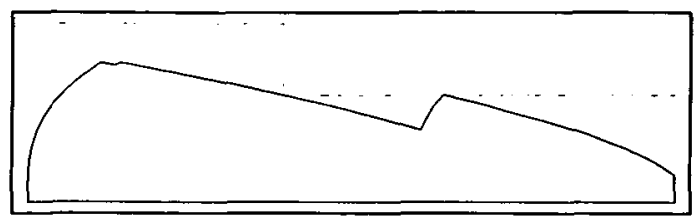

\begin{tabular}{|rrrl|}
\hline time & distance & spoed & control \\
\hline 0.00 & 0.00 & 0.0000 & Power \\
148.89 & 1978.96 & 19.4932 & Coast \\
170.83 & 2402.48 & 19.1022 & Power \\
177.36 & 2528.40 & 19.4932 & Coast \\
436.98 & 7000.00 & 15.0000 & Coast \\
742.00 & 10809.10 & 10.0212 & Power \\
790.22 & 11421.73 & 15.0000 & Coast \\
1496.25 & 17992.88 & 3.8013 & Brake \\
1500.00 & 18000.00 & 0.0000 & \\
& & & \\
\end{tabular}

EXAMPLE 1. 2 intervals, 7 phases

\section{Examples}

In the following examples, the vehicle acceleration is given by

$$
\phi_{u}(v)=\left\{\begin{array}{cl}
1.5 / v & u=\text { power } \\
0 & u=\text { coast } \\
-1 & u=\text { brake }
\end{array}\right.
$$

and resistance is given by

$$
r(v)=0.015+0.00003 v+0.000006 v^{2}
$$

In all examples we use coast-power pairs to approximate speed holding. The number of pairs in each speed limit interval is specified.

In examples 1-3 the track is of length $X=18000$ metres and the required journey time is $T=1500$ seconds. The speed limit for the track is

$$
M(x)= \begin{cases}25 & x<7000 \\ 15 & x \leq 7000\end{cases}
$$

For Example 1, there is one coast-power pair in the first interval and one in the second. The optimal journey has Lagrange multipliers $\mu=0.097400, \lambda_{1}=0.022861$ and $\lambda_{2}=0.025623$. The cost of the journey is $\mathscr{J}=203.63$.

For Example 2, there are no coast-power pairs in the first interval and two in the second. The optimal journey has Lagrange multipliers $\mu=0.065715, \lambda_{1}=$ 0.0212728 and $\lambda_{2}=0.0214888$. The cost of the journey is $\mathscr{J}=202.52$. Note that this journey has the same number of phases as the journey in Example 1, but is less expensive.

Without speed limits, the minimum cost for a journey with seven phases is $\mathscr{J}=$ 201.98. 


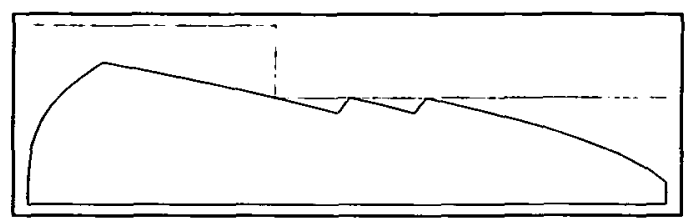

\begin{tabular}{|rrrl|}
\hline time & distance & speed & control \\
\hline 0.00 & 0.00 & 0.0000 & Power \\
155.45 & 2108.15 & 19.8757 & Coast \\
436.42 & 7000.00 & 15.0000 & Coast \\
565.66 & 8799.52 & 12.8568 & Power \\
589.20 & 9128.13 & 15.0000 & Coast \\
718.44 & 10927.65 & 12.8568 & Power \\
741.97 & 11256.26 & 15.0000 & Coast \\
1496.98 & 17995.39 & 3.0581 & Brake \\
1500.00 & 18000.00 & 0.0000 & \\
\hline
\end{tabular}

EXAMPLE 2. 2 intervals, 7 phases

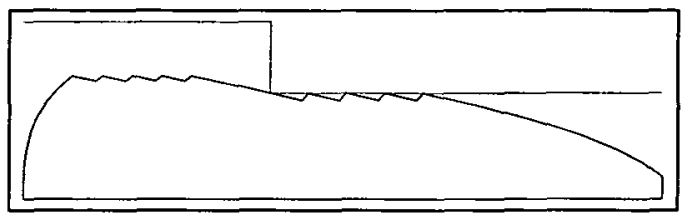

\begin{tabular}{|rrrl|}
\hline time & distance & speed & control \\
\hline 0.00 & 0.00 & 0.0000 & Power \\
115.57 & 1364.00 & 17.3692 & Coast \\
155.59 & 2045.34 & 16.6788 & Power \\
165.34 & 2211.25 & 17.3692 & Coast \\
205.36 & 2892.60 & 16.6788 & Power \\
215.11 & 3058.50 & 17.3692 & Coast \\
255.13 & 3739.85 & 16.6788 & Power \\
264.87 & 3905.76 & 17.3692 & Coast \\
304.90 & 4587.10 & 16.6788 & Power \\
314.64 & 4753.01 & 17.3692 & Coast \\
453.53 & 7000.00 & 15.0000 & Coast \\
515.47 & 7896.96 & 13.9661 & Power \\
527.37 & 8069.44 & 15.0000 & Coast \\
589.30 & 8966.40 & 13.9661 & Power \\
601.21 & 9138.88 & 15.0000 & Coast \\
663.14 & 10035.84 & 13.9661 & Power \\
675.05 & 10208.31 & 15.0000 & Coast \\
736.98 & 11105.27 & 13.9661 & Power \\
748.89 & 11277.75 & 15.0000 & Coast \\
1496.88 & 17995.07 & 3.1644 & Brake \\
1500.00 & 18000.00 & 0.0000 & \\
& & & \\
\hline
\end{tabular}

EXAMPLE 3. 2 intervals, 19 phases

For Example 3, there are four coast-power pairs in each track interval. The optimal journey has Lagrange multipliers $\mu=0.067873, \lambda_{1}=0.021239$ and $\lambda_{2}=0.021449$. The cost of the journey is $\mathscr{J}=202.16$. Without speed limits, the journey cost is $\mathscr{J}=201.89$.

In Examples 4 and 5 the track is also of length $X=18000$ metres, but therequired journey time is $T=1620$ seconds. The speed limit for the track is

$$
M(x)=\left\{\begin{array}{lr}
20 & \\
10 & x<9000 \\
15 & 9000 \leq x \leq 10000 \\
& x>10000 .
\end{array}\right.
$$

For Example 4, there is one coast-power pair in the first interval, two in the second, and one in the third. The optimal journey has Lagrange multipliers $\mu=0.060353$, $\lambda_{1}=0.020798, \lambda_{2}=0.022309$ and $\lambda_{3}=0.020866$. The cost of the journey is $\mathscr{J}=199.14$. Without speed limits, the cost of the journey is $\mathscr{J}=198.01$. 


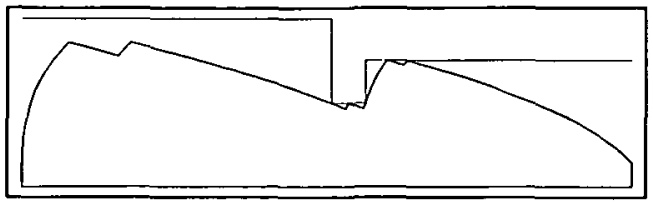

\begin{tabular}{|rrrl|}
\hline time & distance & speed & control \\
\hline 0.00 & 0.00 & 0.0000 & Power \\
112.28 & 1307.18 & 17.1400 & Coast \\
204.42 & 2813.64 & 15.5648 & Power \\
225.53 & 3159.21 & 17.1400 & Coast \\
657.52 & 9000.00 & 10.0000 & Coast \\
704.16 & 9449.10 & 9.2610 & Power \\
709.44 & 9500.00 & 10.0000 & Coast \\
756.08 & 9949.10 & 9.2610 & Power \\
761.36 & 10000.00 & 10.0000 & Power \\
809.74 & 10614.22 & 15.0000 & Coast \\
844.40 & 11124.04 & 14.4198 & Power \\
851.20 & 11224.20 & 15.0000 & Coast \\
1617.15 & 17995.88 & 2.8925 & Brake \\
1620.00 & 18000.00 & 0.0000 & \\
& & & \\
\hline
\end{tabular}

EXAMPLE 4. 3 intervals, 11 phases

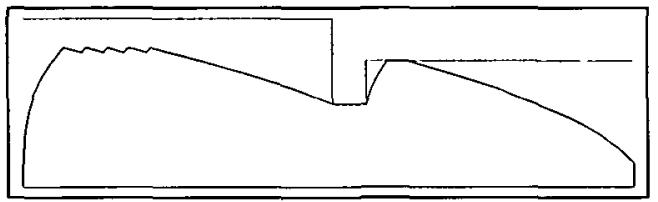

\begin{tabular}{|c|c|c|c|}
\hline time & distance & speed & control \\
\hline 0.00 & 0.00 & 0.0000 & Power \\
\hline 104.14 & 1170.07 & 16.5555 & Coast \\
\hline 136.18 & 1691.67 & 16.0084 & Power \\
\hline 143.47 & 1810.39 & 16.5555 & Coast \\
\hline 175.50 & 2331.98 & 16.0084 & Power \\
\hline 182.79 & 2450.71 & 16.5555 & Coast \\
\hline 214.83 & 2972.30 & 16.0084 & Power \\
\hline 222.12 & 3091.02 & 16.5555 & Coast \\
\hline 254.16 & 3612.62 & 16.0084 & Power \\
\hline 261.45 & 3731.34 & 16.5555 & Coast \\
\hline 659.48 & 9000.00 & 10.0000 & Coast \\
\hline 677.66 & 9179.13 & 9.7114 & Power \\
\hline 679.77 & 9200.00 & 10.0000 & Cosst \\
\hline 697.95 & 9379.13 & 9.7114 & Power \\
\hline 700.07 & 9400.00 & 10.0000 & Coast \\
\hline 718.24 & 9579.13 & 9.7114 & Power \\
\hline 720.36 & 9600.00 & 10.0000 & Coast \\
\hline 738.53 & 9779.13 & 9.7114 & Power \\
\hline 740.65 & 9800.00 & 10.0000 & Coast \\
\hline 758.83 & 9979.13 & 9.7114 & Power \\
\hline 760.94 & 10000.00 & 10.0000 & Power \\
\hline 809.32 & 10614.22 & 15.0000 & Coast \\
\hline 817.78 & 10740.48 & 14.8580 & Power \\
\hline 819.48 & 10765.80 & 15.0000 & Coast \\
\hline 827.93 & 10892.06 & 14.8580 & Power \\
\hline 829.63 & 10917.39 & 15.0000 & Coast \\
\hline 838.09 & 11043.65 & 14.8580 & Power \\
\hline 839.78 & 11068.97 & 15.0000 & Coast \\
\hline 848.24 & 11195.24 & 14.8580 & Power \\
\hline 849.94 & 11220.56 & 15.0000 & Coast \\
\hline 1617.17 & 17995.93 & 2.8731 & Brake \\
\hline 1620.00 & 18000.00 & 0.0000 & \\
\hline
\end{tabular}

EXAMPLE 5. 3 intervals, 29 phases

For Example 5, there are four coast-power pair in the first interval, five in the second, and four in the third. The optimal journey has Lagrange multipliers $\mu=0.059733$, $\lambda_{1}=0.020749, \lambda_{2}=0.022008$ and $\lambda_{3}=0.020791$. The cost of the journey is $\mathscr{J}=199.05$. Without speed limits, the cost of the journey is $\mathscr{J}=197.95$. 


\section{Conclusions}

For a train with three control levels (power, coast and brake) on a straight level track, we can construct an optimal journey. For each given journey time there is a unique holding speed. Speed holding can be approximated by a sequence of coastpower pairs. On intervals of track where the speed limit is below the desired holding speed, the speed must be held at the limit. If braking is necessary on an interval, the speed at which braking commences is determined in part by the holding speed for the interval. We have used the examples to show that the key equations (12) and (13) can be used to determine optimal driving strategies.

\section{References}

[1] I. A. Asnis, A. V. Dmitruk and N. P. Osmolovskii, "Solution of the problem of the energetically optimal control of the motion of a train by the maximum principle", USSR Computational Mathematics and Mathematical Physics 25 (1985) 37-44.

[2] B. R. Benjamin, A. M. Long, I. P. Milroy, R. L. Payne and P. J. Pudney, "Control of railway vehicles for energy conservation and improved timekeeping", Proc. of the Conference on Railway Engineering, 1987.

[3] P. G. Howlett, P. J. Pudney and B. R. Benjamin, "Determination of optimal driving strategies for the control of a train", (to appear), Proc. CTAC 91.

[4] Phil Howlett, "An optimal strategy for the control of a train", J. Austral. Math. Soc., Ser. B 31 (1990) 454-471.

[5] Phil Howlett, "Optimal strategies for the control of a train on a track with non-constant gradient", (submitted), 1992.

[6] Cheng Jaixin and Phil Howlett, "A note on the calculation of optimal strategies for the minimisation of fuel consumption in the control of trains", (to appear), IEEE Transactions on Automatic Control.

[7] Cheng Jiaxin and Phil Howlett, "Application of critical velocities to the minimisation of fuel consumption in the control of trains", Automatica 28 (1) (1992) 165-169.

[8] K. H. Kraft and E. Schnieder, "Optimale trajektorien im spurgebundenen schnellverkehr (optimal trajectories for rapid transit systems)", in Regelungstechnik 29, Jahrgang, 1981).

[9] I. P. Milroy, “Aspects of automatic train control”, Ph. D. Thesis, Loughborough University, 1980. 